\title{
How does back muscle strength change after posterior lumbar interbody fusion?
}

\author{
Chong-Suh Lee, MD, ${ }^{1}$ Kyung-Chung Kang, MD, ${ }^{2}$ Sung-Soo Chung, MD, ${ }^{1}$ Won-Hah Park, MD, ${ }^{3}$ \\ Won-Ju Shin, MD, ${ }^{2}$ and Yong-Gon Seo ${ }^{4}$
}

\begin{abstract}
Departments of ${ }^{1}$ Orthopedic Surgery and ${ }^{3}$ Physical Medicine and Rehabilitation; ${ }^{4}$ Division of Sports Medicine, Department of Physical Medicine and Rehabilitation, Samsung Medical Center, Sungkyunkwan University School of Medicine; and ${ }^{2}$ Department of Orthopedic Surgery, Kyung Hee University Hospital, Kyung Hee University, Seoul, Republic of Korea
\end{abstract}

\begin{abstract}
OBJECTIVE There is a lack of evidence of how back muscle strength changes after lumbar fusion surgery and how exercise influences these changes. The aim of this study was to evaluate changes in back muscle strength after posterior lumbar interbody fusion (PLIF) and to measure the effects of a postoperative exercise program on muscle strength and physical and mental health outcomes.

METHODS This prospective study enrolled 59 women (mean age 58 years) who underwent PLIF at 1 or 2 spinal levels. To assess the effects of a supervised lumbar stabilization exercise (LSE), the authors allocated the patients to an LSE (n $=26)$ or a control $(n=33)$ group. The patients in the LSE group performed the LSEs between 3 and 6 months postoperatively. Back extensor strength, visual analog scale (VAS) scores in back pain, and physical component summary (PCS) and mental component summary (MCS) scores on the 36-Item Short Form Health Survey were determined for the both groups.
\end{abstract}

RESULTS Mean strength of the back muscles tended to slightly decrease by $7.5 \%$ from preoperatively to 3 months after PLIF $(p=0.145)$, but it significantly increased thereafter and was sustained until the last follow-up $(38.1 \%, p<0.001)$. The mean back muscle strength was similar in the LSE and control groups preoperatively, but it increased significantly more in the LSE group (64.2\%) than in the control group (21.7\%) at the last follow-up 12 months after PLIF $(p=0.012)$. At the last follow-up, decreases in back pain VAS scores were more significant among LSE group patients, who had a pain reduction on average of $58.2 \%$, than among control group patients (reduction of $26.1 \%)(p=0.013)$. The patients in the LSE group also had greater improvement in both PCS (39.9\% improvement) and MCS (20.7\% improvement) scores than the patients in the control group (improvement of $18.0 \%$ and $1.1 \%, p=0.042$ and $p=0.035$, respectively).

CONCLUSIONS After PLIF, strength in back muscles decreased until 3 months postoperatively but significantly increased after that period. The patients who regularly underwent postoperative LSE had significantly improved back strength, less pain, and less functional disability at 12 months postoperatively.

https://thejns.org/doi/abs/10.3171/2016.7.SPINE151132

KEY WORDS back muscle strength; change; posterior lumbar interbody fusion; stabilization exercise; lumbar

$\mathrm{B}$ ACK muscle strength is one of the most important factors influencing low-back pain and functional disability. $11,13,15,19$ Particularly for patients with symptomatic lumbar degenerative diseases managed with spinal surgery, it is critical to understand changes in back muscle strength and to improve it after surgery. Choi et al. ${ }^{6}$ reported that patients who had undergone an operation for a herniated lumbar disc and participated in a lumbar extension exercise program had significant improvements in lumbar extensor power and size. A study by Kim et al. ${ }^{21}$ also showed that postoperative training improved lumbar extension strength and disability among patients recovering from lumbar discectomy. However, detailed information is lacking about changes in back muscle strength after lumbar fusion surgery and about the quantitative effects of postoperative exercise programs.

ABBREVIATIONS LSE = lumbar stabilization exercise; $M C S=$ mental component summary; PCS = physical component summary; PLIF = posterior lumbar interbody fusion; QOL = quality of life; SF-36 $=36$-Item Short Form Health Survey; VAS = visual analog scale.

SUBMITTED September 22, 2015. ACCEPTED July 18, 2016.

INCLUDE WHEN CITING Published online October 14, 2016; DOI: 10.3171/2016.7.SPINE151132. 
Patients who undergo lumbar spinal fusion have a larger amount of muscle denervation, a longer retraction time during the operation, and a longer period of postoperative immobilization than patients who undergo simple lumbar discectomy or decompression. Therefore, patients who underwent lumbar fusion have greater weakness or more severe damage to the back muscles., ${ }^{9,17,18}$ Although some studies have evaluated the changes of trunk muscle strength after lumbar fusion surgery, their findings have been incomplete. For example, Tarnanen et al..$^{33}$ reported that patients who underwent lumbar fusion have low trunk extension and flexion strength levels at 3 months postoperatively, but the authors also noted an early change in these variables at this time and did not examine any changes after that 3-month period. Using measurements of the paraspinal muscle activity, the authors analyzed the results of rehabilitation after lumbar spinal fusion, but they did not report on any specific changes in muscle strength after the operation..$^{32}$

To date and to the best of our knowledge, no study has comprehensively evaluated changes in back muscle strength after posterior lumbar interbody fusion (PLIF). Furthermore, because no standard back exercise programs after lumbar fusions have yet been developed, ${ }^{31}$ understanding the changes in back muscle strength after PLIF and the effects of appropriate postoperative exercises on muscle strength likely have important roles for better surgical outcomes. Therefore, the aim of this study was to evaluate changes in back muscle strength after lumbar spinal fusion and the effects of a postoperative exercise program on these changes.

\section{Methods}

\section{Study Protocols and Patients}

The present study was prospectively designed to evaluate changes in back muscle strength after PLIF and to measure the effects of a postoperative lumbar stabilization exercise (LSE). The study protocol was approved by the Samsung Medical Center institutional review board, and informed consent from each participant was obtained before the study was commenced.

Sixty-five women with symptomatic lumbar degenerative diseases were recruited from the Department of Orthopedic Surgery at Samsung Medical Center between January 2010 and December 2010. The mean age was 58 years (range 43-68 years). The inclusion criteria stipulated that adequate nonsurgical treatments had failed to improve back pain and that patients had functional disability for at least 6 months, that they were candidates for 1- or 2-level PLIF between L-3 and S-1, and that they were available for complete follow-up visits that included assessments with quality of life (QOL) questionnaires. The exclusion criteria were the following: 1) a previous lumbar spinal operation, 2) a combined cervical or thoracic myelopathy, 3) an inability to respond to questions in the questionnaires and to properly follow the protocol of the strength test, and 4) a failure to perform activities of daily living because of comorbidities (e.g., cerebrovascular accident, Parkinson disease, psychotic disorders, or other orthopedic degenerative diseases). Men were not included in
TABLE 1. Baseline demographics of patients in the exercise and control groups

\begin{tabular}{lccc}
\hline \multirow{2}{*}{ Variable } & \multicolumn{2}{c}{ Patient Group } & $\begin{array}{c}p \\
\text { Value* }\end{array}$ \\
\cline { 2 - 3 } No. of patients & 26 & 33 & NA \\
\hline Mean age in yrs & $55 \pm 4.8$ & $59 \pm 5.1$ & 0.118 \\
\hline Mean weight in kg & $54.4 \pm 6.2$ & $57.8 \pm 7.7$ & 0.304 \\
\hline BMl in kg/m ${ }^{2}$ & $22.2 \pm 2.1$ & $23.1 \pm 2.4$ & 0.620 \\
\hline Mean BMD T-score & $-1.24 \pm 0.55$ & $-1.66 \pm 0.61$ & 0.311 \\
\hline Mean current smoking & 3 & 2 & 0.453 \\
\hline Alcohol use & 4 & 3 & 0.458 \\
\hline Diabetes & 4 & 5 & 0.980 \\
\hline Steroid use & 2 & 2 & 0.805 \\
\hline No. of fused spinal levels (\%) & & & 0.870 \\
\hline \multicolumn{1}{c}{ 1 level } & $19(73.1)$ & $24(72.7)$ & \\
\hline 2 levels & $7(26.9)$ & $9(27.3)$ & \\
\hline
\end{tabular}

$\mathrm{BMD}=$ bone mineral density; $\mathrm{BMI}=$ body mass index $\mathrm{NA}=$ not applicable.

Mean values are presented \pm SD.

* A p value of $<0.05$ was considered statistically significant.

the present study because only a small number of potential participants were available, and there was the possibility of lower participation by men in clinic-based supervised postoperative exercises.

All diagnoses and procedures were performed by 1 senior surgeon at our institution (C.S.L.). In all cases, PLIF with a titanium cage and concomitant decompression were performed and augmented by posterior spinal instrumentation. When possible, an autogenous local bone graft obtained during surgery was used in the fusion, and an irradiated allograft bone was also used if insufficient local bone was available. We did not use bone morphogenetic protein, demineralized bone matrix, or any other kinds of bone substitutes in this study. For 3 months postoperatively, all patients wore a rigid lumbosacral orthosis.

\section{Postoperative LSE}

Initially, all patients were recommended to join a postoperative LSE program. Among them, 30 patients were permitted to participate in the postoperative LSE (representing the LSE group) and 35 patients were not (i.e., were included in a control group). In total, 4 patients were excluded from further analysis because of an incomplete strength test during the follow-up period, and 2 patients in the LSE group did not complete the LSE program (i.e., they completed $<90 \%$ of the LSE). The data from the remaining 59 patients were analyzed in this study. The LSE and control groups did not significantly differ in demographic characteristics (Table 1). The 26 patients in the LSE group comprised 9 patients with combined degenerative disc disease and spinal stenosis, 10 with degenerative spondylolisthesis, and 7 with spondylolytic spondylolisthesis. The respective order of the 33 patients in the control group was 9,14 , and 10 patients.

At 3 months after PLIF, patients in the LSE group were instructed in an exercise protocol developed at our institution. The clinic-based LSE consisted of 36 sessions of 

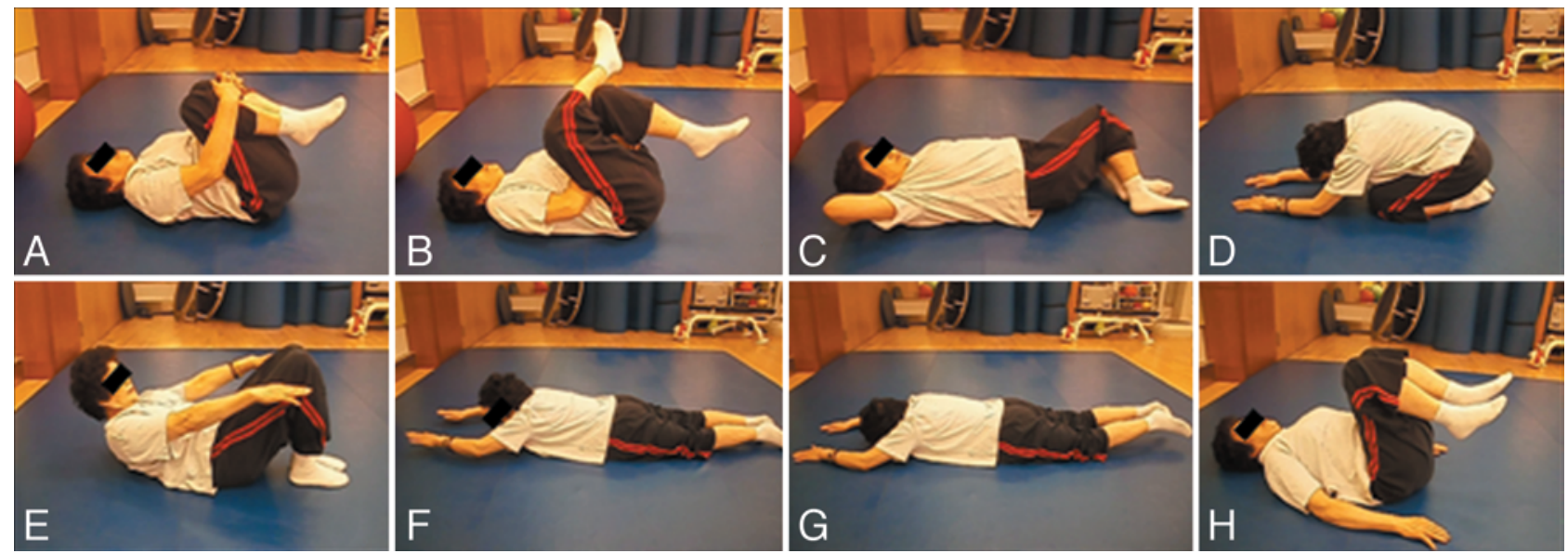

FIG. 1. Examples of the postoperative LSE program. A 62-year-old patient who underwent L4-S1 PLIF started the exercises at 3 months postoperatively. The LSE was performed without a range-of-motion restriction of the lumbar spine and involved stretching and strengthening exercises, including knee to chest $(\mathbf{A})$, piriformis $(\mathbf{B})$, lower body twist $(\mathbf{C})$, praying and strengthening position $(D)$, partial sit-up $(E)$, prone arms raise $(\mathbf{F})$, prone legs raise $(\mathbf{G})$, and reverse crunch $(\mathbf{H})$. Figure is available in color online only.

1 hour each and ran for 12 weeks. The LSE consists of a series of warm-up and main exercises of chiefly back stretching and lumbar stabilization ${ }^{2,3,24,26}$ and is performed without a range-of-motion restriction in the lumbar spine. The warm-up exercises were performed for 15 minutes on a treadmill or bicycle, and the main exercise was closely supervised by 1 physical therapist (Y.G.S.) at our sports medicine center. The main exercise postures are shown in Fig. 1 and were divided into stretching positions (knee to chest, modified knee to chest, piriformis, lower body twist, cat and camel position, and prayer position) and strengthening positions (pelvic tilt, partial sit-up, bridge, prone arms and legs raise, and reverse crunch). The 33 patients in the control group had no exercise prescription and were asked to continue their usual exercise and physical activities without any restriction.

\section{Clinical Parameters}

The primary outcome measure was back muscle strength measured as maximal isometric strength. Secondary outcome measures were intensity of back pain, assessed with a $100-\mathrm{mm}$ visual analog scale (VAS), and functional disability, evaluated with questionnaires on health-related QOL with the 36-Item Short Form Health Survey (SF-36). Physical health scores were determined with the physical component summary (PCS) and mental health scores with the mental component summary (MCS) of the SF-36. Data were collected prospectively before surgery and at 3,6, and 12 months after surgery.

The isometric strength of back muscles was determined with a lumbar extension machine (MedX). Each test measured the maximal voluntary isometric strength of the lumbar extensor muscles at 7 angular positions $\left(0^{\circ}, 12^{\circ}\right.$, $24^{\circ}, 36^{\circ}, 48^{\circ}, 60^{\circ}$, and $72^{\circ}$ ) of lumbar flexion. All patients were thoroughly instructed in the method for accurate testing and performed warm-up exercises for 15 minutes before the test. The patients were positioned according to an established protocol and were then asked to increase the lumbar extension torque over a 2- to 3-second period. Once maximal tension had been achieved, the patients were instructed to maintain the contraction for an additional 1-2 seconds. After reaching maximum torque, they were asked to slowly decrease the torque. During the contractions, visual feedback was provided on a video display screen interfaced with the machine, and the patients were also verbally encouraged to give their maximum effort..$^{10,25}$ The patients were given a 10 -second resting period between angle conditions, and the maximal isometric voluntary extension of the back muscles was measured (in footpounds). All patients were assessed by the same physical therapist at our sports medicine center who was blinded to other results. Repeated tests for measuring reliability were not conducted.

The body mass index and bone density were also measured. Interbody fusion was defined as an angulation of $5^{\circ}$ or less and translation as $3 \mathrm{~mm}$ or less on full flexionextension lateral radiographs and as continuous trabecular bone growth connecting the vertebral bodies assessed on reformatted CT scans at 12 months postoperatively. ${ }^{5}$

\section{Statistical Analysis}

With a predefined statistical significance level of 5\% and to achieve a statistical power of $80 \%$, we calculated that the minimum sample size needed for this study was 60 evaluable patients. ${ }^{21}$ A medical statistical consultant performed the statistical analyses with SAS version 9.1 (SAS Institute Inc.). The data were expressed as mean \pm SD and were analyzed with paired and unpaired Student t-tests, Mann-Whitney U-test, and Wilcoxon signed-rank test. A p $<0.05$ was considered statistically significant.

\section{Results}

\section{Changes of Back Extensor Strength After PLIF}

In the 59 patients evaluated in the present study, preoperative mean extensor strengths $(95.6 \pm 27.2 \mathrm{ft}-\mathrm{lb})$ gradually decreased by $7.5 \%$ until 3 months after PLIF $(88.4 \pm$ $27.6 \mathrm{ft}-\mathrm{lb}$ ), but this decrease was not statistically significant $(\mathrm{p}=0.145)$. Compared with preoperative extensor strengths, the mean strengths significantly increased by $29.7 \%$ at 6 
months postoperatively $(124.0 \pm 34.4 \mathrm{ft}-\mathrm{lb})(\mathrm{p}<0.001)$, and these strengths were maintained until the last follow-up at 12 months postoperatively $(132.0 \pm 32.4 \mathrm{ft}-\mathrm{lb}$, corresponding to a $38.1 \%$ increase) $(\mathrm{p}<0.001)$. At 12 months postoperatively, extensor strength slightly increased, but the increase between 6 and 12 months postoperatively was not statistically significant $(\mathrm{p}=0.171)($ Fig. 2$)$.

\section{Effects of Postoperative LSE on Muscle Strength and Back Pain}

No adverse effects of the LSE were observed. The LSE and control groups had similar extensor strengths both preoperatively (LSE group $90.5 \pm 26.8 \mathrm{ft}-\mathrm{lb}$ vs control group $99.1 \pm 27.7 \mathrm{ft}-\mathrm{lb}, \mathrm{p}=0.468)$ and 3 months postoperatively (LSE group $83.4 \pm 25.6 \mathrm{ft}-\mathrm{lb}$ vs control group $91.5 \pm 29.2 \mathrm{ft}-\mathrm{lb}, \mathrm{p}=0.488$ ). However, after completion of the 12-week LSE program at 6 months postoperatively, the patients in the LSE group had significantly higher mean extensor strength $(135.1 \pm 24.9 \mathrm{ft}-\mathrm{lb}$, corresponding to a $49.3 \%$ increase relative to the preoperative strength) than those in the control group $(116.4 \pm 38.6 \mathrm{ft}-\mathrm{lb}$, corresponding to a $17.5 \%$ increase relative to the preoperative strength) (p $=0.012$ ). At 12 months postoperatively, the mean strength was also significantly higher in the LSE group (148.6 \pm $23.6 \mathrm{ft}-\mathrm{lb}$, corresponding to a $64.2 \%$ increase relative to preoperative strength) than in the control group (120.6 \pm $33.1 \mathrm{ft}-\mathrm{lb}$, corresponding to a $21.7 \%$ increase relative to the preoperative strength $)(\mathrm{p}=0.012)$.

In both groups, the mean preoperative VAS score for back pain (LSE group $58.6 \pm 26.9$ and control group 59.5 \pm 26.7 ) had decreased significantly by the last follow-up at 12 months postoperatively (LSE group $24.5 \pm 14.4$ and control group $44.0 \pm 21.9)(\mathrm{p}=0.004$ and $\mathrm{p}=0.031$, respectively). However, the rate of the decrease in self-reported back pain was more significant in the LSE group than in the control group at 6 months postoperatively (decrease of $56.3 \%$ and $29.6 \%$, respectively, $\mathrm{p}=0.021$ ) and at the last follow-up (decrease of $58.2 \%$ and $26.1 \%$ respectively, $\mathrm{p}=$ 0.013) (Fig. 3).

\section{Effects of Postoperative LSE on Physical and Mental Health}

For physical health scores, we noted that PCS scores of both groups (mean preoperative scores for the LSE group $31.6 \pm 4.8$ and for the control group $32.3 \pm 6.1$ ) had significantly improved by the last follow-up at 12 months postoperatively (LSE group $44.2 \pm 5.2$ and control group $38.1 \pm$ 8.7) ( $\mathrm{p}<0.001$ and $\mathrm{p}=0.014$, respectively). The mean preoperative MCS score in the exercise group (37.6 \pm 7.3$)$ had significantly improved by the last follow-up $(45.4 \pm 9.5)(\mathrm{p}$ $=0.006$ ), but the MCS score in the control group (36.6 \pm 11.9) did not significantly improve from preoperatively to the last follow-up $(37.0 \pm 11.1)(\mathrm{p}=0.883)$. The LSE group had statistically greater improvements in the PCS score (39.9\% improvement) and the MCS score (20.7\% improvement) than the control group (improvement of $18.0 \%$ and $1.1 \%, p=0.042$ and $\mathrm{p}=0.035$, respectively) (Fig. 4).

Preoperatively, scores on the 8 sections of the SF-36that is, for physical functioning, physical role functioning, general health perceptions, vitality, social role functioning, emotional role functioning, and mental health-did

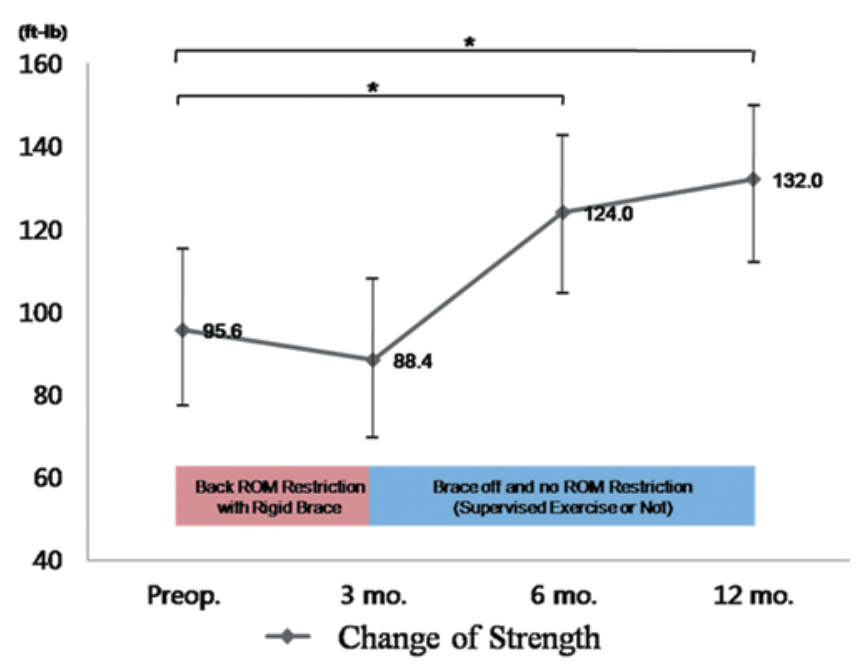

FIG. 2. Changes in back extensor strength in all patients. Compared with preoperative back muscle strength, extensor strength gradually decreased by $7.5 \%$ until 3 months after PLIF but then significantly increased by $29.7 \%$ at 6 months and by $38.1 \%$ at 12 months postoperatively. Data shown as the mean \pm SD; the brackets with asterisks indicate statistically significant differences in back extensor strength $(p<$ $0.05)$. ROM = range of motion. Figure is available in color online only.

not significantly differ between the LSE and control groups ( $p>0.05$ ), except for bodily pain (LSE group 36.5 \pm 5.8 and control group $31.4 \pm 6.5, \mathrm{p}=0.038$ ). However, at the last follow-up, the scores for physical role functioning, bodily pain, general health perception, vitality, and social functioning were significantly higher in the exercise group than in the control group ( $<<0.05)$ (Fig. 5).

\section{Radiographic Results}

None of the patients showed an angulation of $5^{\circ}$ or greater or a translation of $3 \mathrm{~mm}$ or more on full flexionextension lateral radiographs. On CT scans acquired 12 months postoperatively, $25(96 \%)$ of the 26 patients in the exercise group and 31 (94\%) of the 33 patients in the control group had complete bone bridge formation between the adjacent vertebral bodies. In each group, patients without a complete bone bridge did not have a continuous bone bridge on their CT scan but also had no radiographic evidence of instability or clinical correlation with nonunion.

\section{Discussion}

In this study, after PLIF of 1 or 2 spinal levels, a clinicbased 12-week LSE significantly improved back extensor muscle strength, low-back pain, functional disability, and health-related QOL scores, without any adverse effects. Mean isometric strengths slightly decreased from preoperatively to 3 months postoperatively and then significantly increased at 6 and 12 months postoperatively in all patients. However, the increase in back strength at the last follow-up was much higher among the patients in the LSE group (64.2\%) than among those in the control group (21.7\%). Moreover, back pain decreased more significantly in the LSE group (58.2\%) than in the control group (26.1\%). Also at the last follow-up, although the PCS and MCS had increased in both groups, the increments of 

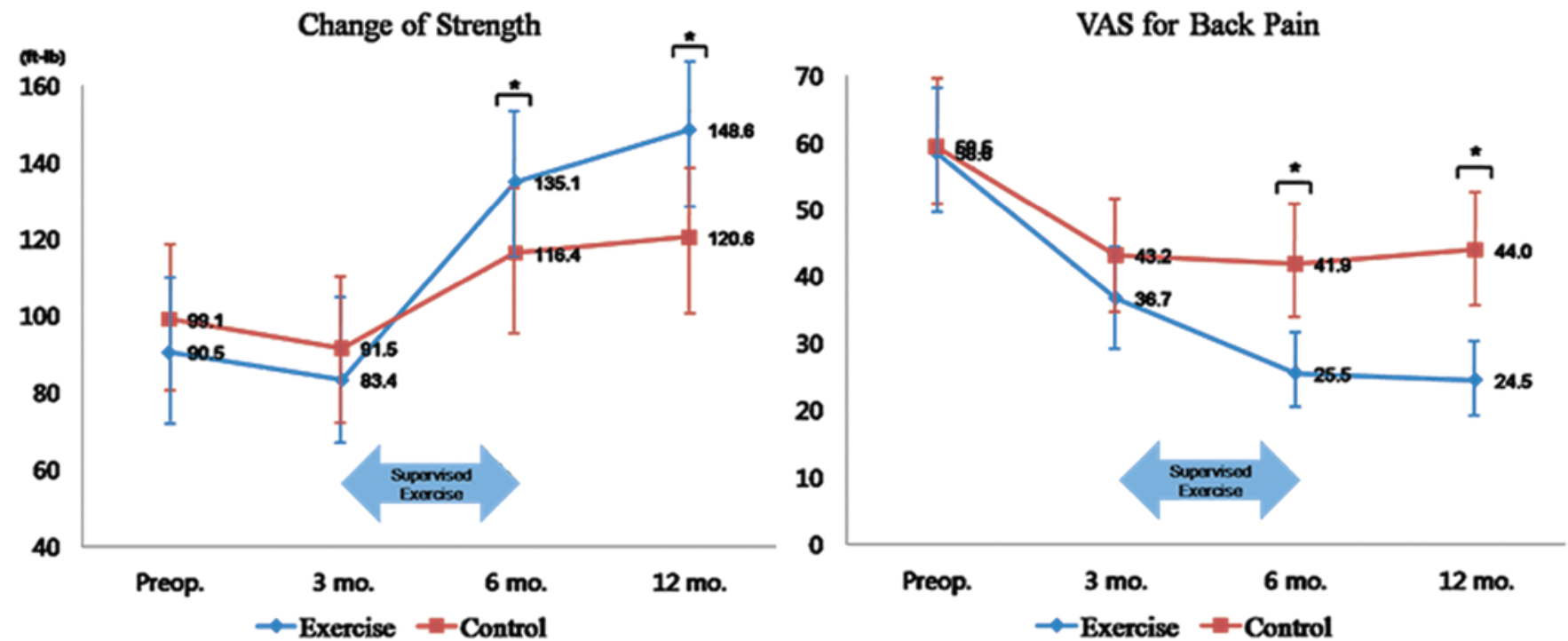

FIG. 3. Back strength and back pain in the LSE and control groups. Supervised LSE was started at 3 months postoperatively and ran for 12 weeks. The preoperative mean extensor strength was similar for both groups, but at the last follow-up 12 months postoperatively, the mean extensor strength had significantly increased by $64.2 \%$ in the LSE group compared with only $21.7 \%$ in the control group (left). Decreases in back pain VAS scores were significantly $(p<0.05)$ larger among patients in the LSE group ( $58.2 \%$ decrease) than among those in the control group ( $26.1 \%$ decrease) (right). Data shown as the mean \pm SD. The brackets with asterisks indicate statistically significant differences between the LSE and control groups at each postoperative follow-up.

Figure is available in color online only.

the PCS and MCS scores were significantly higher for the patients in the LSE group (i.e., an increase of $39.9 \%$ and $20.7 \%$, respectively) than the patients in the control group (increases of $18.0 \%$ and $1.1 \%$, respectively).

In general, back muscle strength is considered one of the most important factors for reducing low-back pain and functional disability and for planning rehabilitation modalities. ${ }^{12,16,20,23,34}$ Furthermore, patients with symptomatic lumbar degenerative diseases that are managed with lumbar fusion surgery commonly show severe deterioration
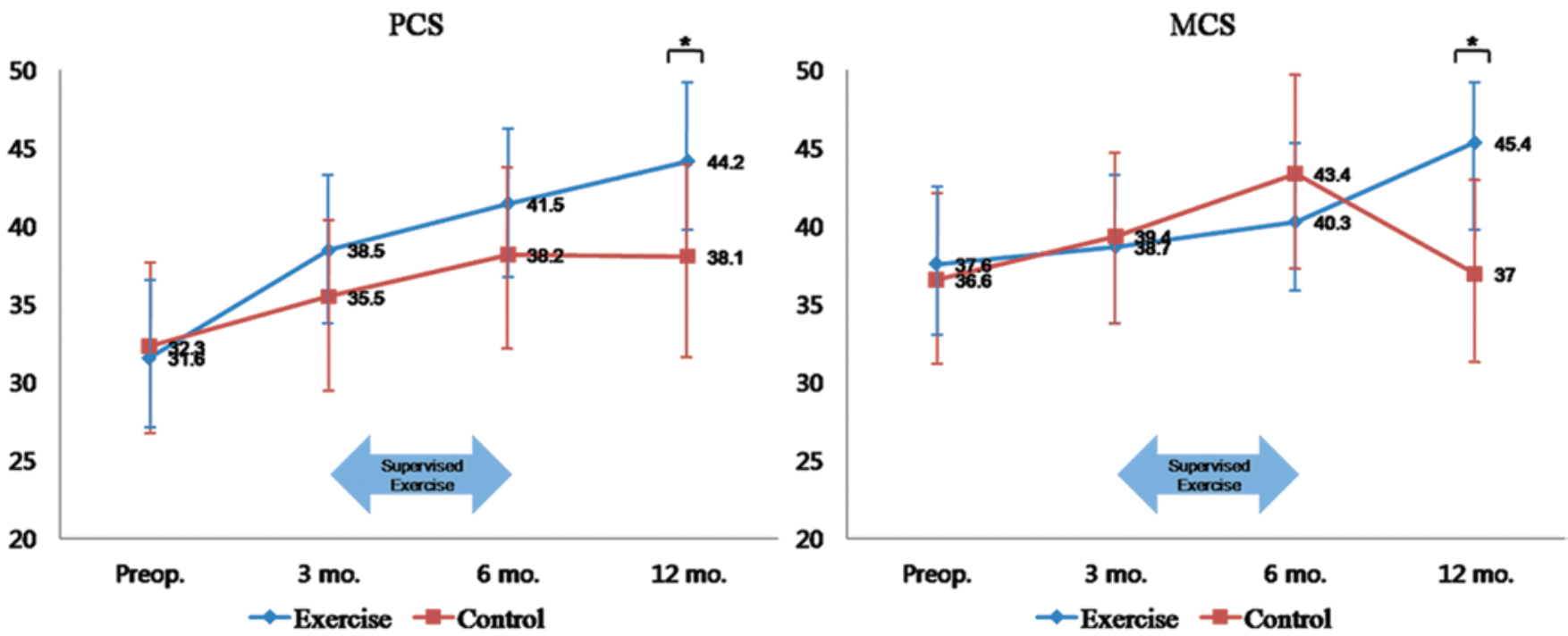

FIG. 4. Changes in PCS and MCS scores in the LSE and control groups from preoperatively to postoperatively. Left: The mean PCS scores of both groups had significantly improved by the last follow-up at 12 months postoperatively $(p<0.05)$. Right: The MCS scores of the LSE group also had improved by the last follow-up, but not the MCS scores of the control group, which were very similar to the preoperative scores $(p=0.883)$. Overall, the patients in the LSE group had significantly higher increment rates in PCS scores (i.e., an increase of 39.9\%) and MCS scores (increase of 20.7\%) than the patients in the control group at 12 months postoperatively (increases of $18.0 \%$ and $1.1 \%, p=0.042$ and $p=0.035$, respectively). The brackets with asterisks indicate statistically significant differences between the LSE and control groups at 12 months postoperatively. Figure is available in color online only. 

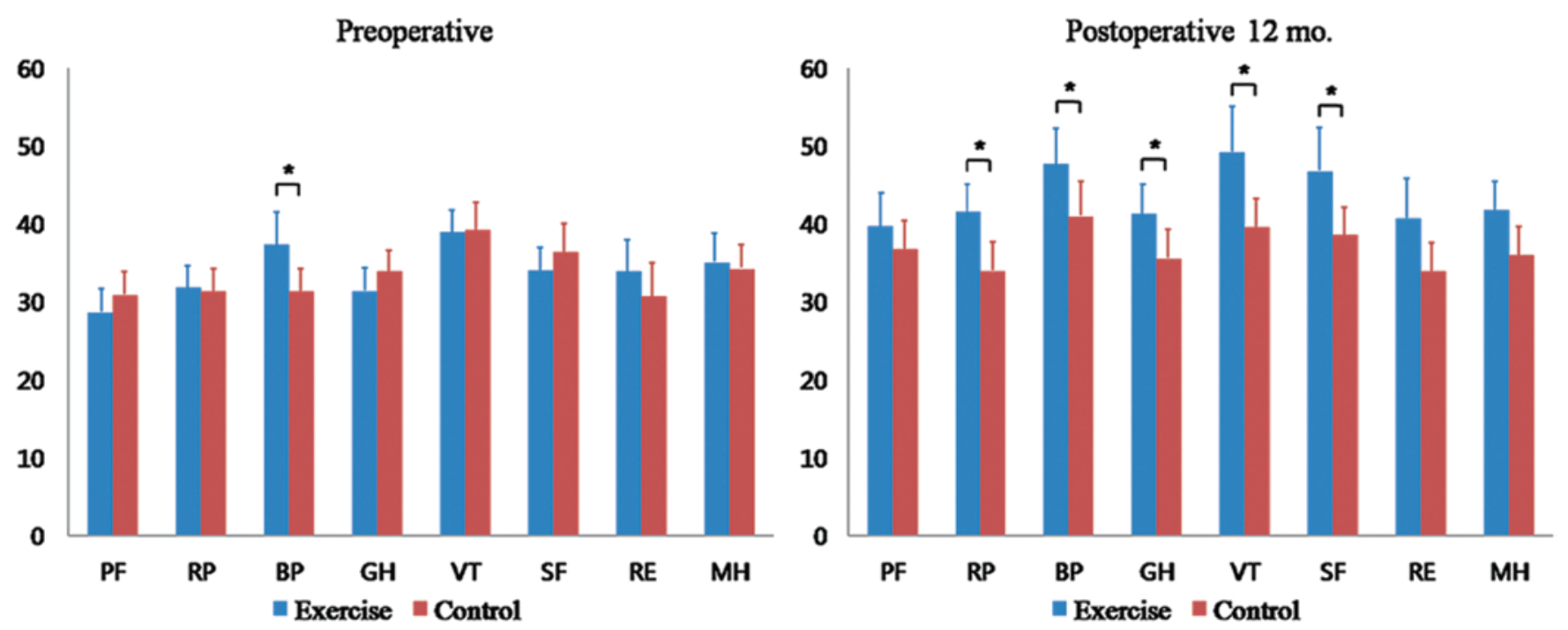

FIG. 5. Intergroup comparisons of the scores on the 8 sections in the SF-36. Preoperative scores for physical functioning, physical role functioning, general health, vitality, social functioning, emotional role functioning, and mental health did not statistically significantly differ between the exercise and control groups $(p>0.05)$, except for bodily pain $(p=0.038)$ (left). At the last follow-up, scores for physical role functioning, bodily pain, general health, vitality, and social functioning were significantly higher among patients in the LSE group than among the control group patients $(p<0.05)$ (right). Brackets with asterisks indicate statistically significant differences. $\mathrm{BP}=$ bodily pain; $\mathrm{GH}=$ general health; $\mathrm{MH}=$ mental health; $\mathrm{PF}=$ physical functioning; $\mathrm{RE}=$ emotional role functioning; RP = physical role functioning; SF = social functioning; VT = vitality. Figure is available in color online only.

in back muscle strength, and their muscle strength further decreases because of muscle damage during surgery and postoperative immobilization with a rigid brace. However, only a few studies have investigated the outcomes of lumbar spinal fusion and the effects of postoperative back exercises on back muscle strength. Two randomized controlled trials have examined the effects of different types of rehabilitation programs after lumbar spinal fusion. Results of studies by Christensen et al. ${ }^{7}$ and Abbott et al. ${ }^{1}$ indicated significantly better outcomes with rehabilitation programs, emphasizing the importance of a psychosocial approach rather than exercise-only aftercare. However, neither study reported on the quantitative effects of postoperative lumbar exercises supervised by a physical therapist. Furthermore, the surgical techniques and instrumentation were not consistently controlled in these previous investigations. Several other reports have highlighted the superiority of clinic-based supervised exercises for patients with chronic low-back pain, but those studies did not examine patients who underwent lumbar spine surgery. $4,8,22$

Although a few studies have reported on the safety and efficacy of early rehabilitation in the 3-month postoperative period, ${ }^{1,28}$ controversies still remain about the timing of rehabilitation or the intensity of back muscle exercises after spinal fusion surgery. Using quantitative tools to measure back extensor strength, pain, and functional disability, we show here that postoperative LSE between Months 3 and 6 after lumbar spinal fusion is safe and efficacious. The authors of previous reports were unable to suggest an appropriate time to exercise after lumbar fusion, but the results of the current study confirm the efficacy and safety of commonly used back-stabilization exercises at 3 months postoperatively in patients who underwent PLIF.

After consultation with a doctor of rehabilitation medi- cine and a physical therapist, all patients in the exercise group were provided with a specific training regimen at our sports medical center. The exercise group was asked to attend 36 sessions (of 1 hour each) and to perform exercises with progressively increasing intensity. The strengthening exercises were supplemented with a short-time aerobic warm-up, and the patients also performed a series of 6 static stretching exercises before the strengthening exercises. Although the repetition or intensity of the exercises was controlled according to each patient's condition or exercise capacity, all patients performed the exercises without a restriction of range of motion in the lumbar spine. The total cost for the LSE program was about $\$ 900$ for each patient, and all patients in this study received financial aid without any conflict of interest.

For patients with symptomatic lumbar diseases, reliable measurements of back muscle strength may be difficult because back pain and discomfort may preclude accurate strength assessments. However, previous studies have supported the reliability of back muscle strength tests, particularly for measuring isometric strength, for elderly people or for patients with chronic low-back pain..$^{14,15,27,29,30}$ In addition, in a recent study, we obtained a satisfactory result, observing high reliability (i.e., an intraclass correlation coefficient of 0.951) with repeated measurements of isometric strength in 1-week intervals of 10 patients (data not shown).

Interestingly, most of the patients in the control group noted on the postoperative questionnaires that they did not exercise except for walking. Although back strength of the control group had increased without the lumbar exercise after 6 months postoperatively, their improvements in strength were not comparable to those of the LSE group. The patients were concerned that the postoperative back exercise may cause back problems in the operated area. 
Indeed, many spine surgeons usually recommend only walking or simple back exercises postoperatively. However, we found no evidence for any associations between complications after PLIF and postoperative exercise, and some studies support the need for systematic back exercise postoperatively to aid patients' recovery. ${ }^{1,31}$ Although the authors of these studies did not use an established postoperative exercise program, they confirmed the efficacy and safety of postoperative back exercises even in patients with spinal fusion surgery.

\section{Study Limitations}

This study had some limitations. First, we did not perform a proper randomization. However, we note that preoperative demographics were very similar between the 2 groups. Although most of the patients wanted to participate in the clinic-based postoperative LSE program, a true randomization was difficult because the program ran for 12 weeks and differences in work obligations and distances between the hospital and patients' residences would have influenced patient participation. Second, back strength was evaluated only as lumbar extensor strength and did not include flexion power of other trunk muscles. Although a balance between the flexion and extension strength is considered to be important in controlling back pain and functional disability, ${ }^{30,32,33}$ we deemed evaluating back extensor strength important for patients with PLIF because this surgical approach mainly has an effect on the deterioration of lumbar extensor muscles, such as the longissimus or multifidus muscles. Last, the patients in the LSE group tended to be younger than the patients in the control group, and younger age might be an important factor influencing postoperative outcomes. Further studies with a longer followup and with a larger patient cohort are therefore needed. Nonetheless, the results of the present study were based on validated and quantitative measurement tools and provide new evidence that LSE is safe and of benefit to patients who underwent lumbar spinal fusion.

\section{Conclusions}

Back extensor strength in patients who underwent PLIF slightly decreased until 3 months postoperatively, but significantly increased after that time. The patients participating in a postoperative LSE program exhibited significant improvements in back strength, reported less back pain, and had less functional disability. However, further study is needed to determine the long-term effects of this exercise program on postoperative recovery of muscle strength after PLIF.

\section{Acknowledgments}

This study was supported by funding from Clinical Research Development Program (grant CRDP CRS110-27-1) to Samsung Medical Center.

\section{References}

1. Abbott AD, Tyni-Lenné R, Hedlund R: Early rehabilitation targeting cognition, behavior, and motor function after lumbar fusion: a randomized controlled trial. Spine (Phila Pa 1976) $35: 848-857,2010$
2. Barr KP, Griggs M, Cadby T: Lumbar stabilization: core concepts and current literature, Part 1. Am J Phys Med Rehabil 84:473-480, 2005

3. Barr KP, Griggs M, Cadby T: Lumbar stabilization: a review of core concepts and current literature, part 2. Am J Phys Med Rehabil 86:72-80, 2007

4. Bronfort G, Maiers MJ, Evans RL, Schulz CA, Bracha Y, Svendsen KH, et al: Supervised exercise, spinal manipulation, and home exercise for chronic low back pain: a randomized clinical trial. Spine J 11:585-598, 2011

5. Burkus JK, Dorchak JD, Sanders DL: Radiographic assessment of interbody fusion using recombinant human bone morphogenetic protein type 2. Spine (Phila Pa 1976) 28:372-377, 2003

6. Choi G, Raiturker PP, Kim MJ, Chung DJ, Chae YS, Lee $\mathrm{SH}$ : The effect of early isolated lumbar extension exercise program for patients with herniated disc undergoing lumbar discectomy. Neurosurgery 57:764-772, 2005

7. Christensen FB, Laurberg I, Bünger CE: Importance of the back-café concept to rehabilitation after lumbar spinal fusion: a randomized clinical study with a 2-year follow-up. Spine (Phila Pa 1976) 28:2561-2569, 2003

8. Friedrich M, Cermak T, Maderbacher P: The effect of brochure use versus therapist teaching on patients performing therapeutic exercise and on changes in impairment status. Phys Ther 76:1082-1088, 1996

9. Gejo R, Matsui H, Kawaguchi Y, Ishihara H, Tsuji H: Serial changes in trunk muscle performance after posterior lumbar surgery. Spine (Phila Pa 1976) 24:1023-1028, 1999

10. Graves JE, Webb DC, Pollock ML, Matkozich J, Leggett SH, Carpenter DM, et al: Pelvic stabilization during resistance training: its effect on the development of lumbar extension strength. Arch Phys Med Rehabil 75:210-215, 1994

11. Häkkinen A, Ylinen J, Kautiainen H, Airaksinen O, Herno A, Tarvainen U, et al: Pain, trunk muscle strength, spine mobility and disability following lumbar disc surgery. J Rehabil Med 35:236-240, 2003

12. Hirsch G, Beach G, Cooke C, Menard M, Locke S: Relationship between performance on lumbar dynamometry and Waddell score in a population with low-back pain. Spine (Phila Pa 1976) 16:1039-1043, 1991

13. Ho CW, Chen LC, Hsu HH, Chiang SL, Li MH, Jiang SH, et $\mathrm{al}$ : Isokinetic muscle strength of the trunk and bilateral knees in young subjects with lumbar disc herniation. Spine (Phila Pa 1976) 30:E528-E533, 2005

14. Hutten MM, Hermens HJ: Reliability of lumbar dynamometry measurements in patients with chronic low back pain with test-retest measurements on different days. Eur Spine J 6:54-62, 1997

15. Imagama S, Matsuyama Y, Hasegawa Y, Sakai Y, Ito Z, Ishiguro N, et al: Back muscle strength and spinal mobility are predictors of quality of life in middle-aged and elderly males. Eur Spine J 20:954-961, 2011

16. Karataş GK, Göğüus F, Meray J: Reliability of isokinetic trunk muscle strength measurement. Am J Phys Med Rehabil 81:79-85, 2002

17. Kawaguchi Y, Matsui $\mathrm{H}$, Tsuji $\mathrm{H}$ : Back muscle injury after posterior lumbar spine surgery. A histologic and enzymatic analysis. Spine (Phila Pa 1976) 21:941-944, 1996

18. Kawaguchi Y, Matsui H, Tsuji H: Back muscle injury after posterior lumbar spine surgery. Part 2: Histologic and histochemical analyses in humans. Spine (Phila Pa 1976) 19:2598-2602, 1994

19. Keller A, Brox JI, Gunderson R, Holm I, Friis A, Reikerås $\mathrm{O}$ : Trunk muscle strength, cross-sectional area, and density in patients with chronic low back pain randomized to lumbar fusion or cognitive intervention and exercises. Spine (Phila Pa 1976) 29:3-8, 2004

20. Keller A, Hellesnes J, Brox JI: Reliability of the isokinetic trunk extensor test, Biering-Sørensen test, and Astrand bi- 
cycle test: assessment of intraclass correlation coefficient and critical difference in patients with chronic low back pain and healthy individuals. Spine (Phila Pa 1976) 26:771-777, 2001

21. Kim YS, Park J, Hsu J, Cho KK, Kim YH, Shim JK: Effects of training frequency on lumbar extension strength in patients recovering from lumbar dyscectomy. J Rehabil Med 42:839-845, 2010

22. Maul I, Läubli T, Oliveri M, Krueger H: Long-term effects of supervised physical training in secondary prevention of low back pain. Eur Spine J 14:599-611, 2005

23. Mayer T, Gatchel R, Betancur J, Bovasso E: Trunk muscle endurance measurement. Isometric contrasted to isokinetic testing in normal subjects. Spine (Phila Pa 1976) 20:920927, 1995

24. O'Sullivan PB, Phyty GD, Twomey LT, Allison GT: Evaluation of specific stabilizing exercise in the treatment of chronic low back pain with radiologic diagnosis of spondylolysis or spondylolisthesis. Spine (Phila Pa 1976) 22:2959-2967, 1997

25. Pollock ML, Leggett SH, Graves JE, Jones A, Fulton M, Cirulli J: Effect of resistance training on lumbar extension strength. Am J Sports Med 17:624-629, 1989

26. Richardson CA, Jull GA: Muscle control-pain control. What exercises would you prescribe? Man Ther 1:2-10, 1995

27. Robinson ME, Greene AF, O'Connor P, Graves JE, MacMillan M: Reliability of lumbar isometric torque in patients with chronic low back pain. Phys Ther 72:186-190, 1992

28. Rohlmann A, Graichen F, Bergmann G: Loads on an internal spinal fixation device during physical therapy. Phys Ther 82:44-52, 2002

29. Roussel NA, Truijen S, De Kerf I, Lambeets D, Nijs J, Stassijns G: Reliability of the assessment of lumbar range of motion and maximal isometric strength in patients with chronic low back pain. Arch Phys Med Rehabil 89:788-791, 2008

30. Scheuer R, Friedrich M: Reliability of isometric strength measurements in trunk and neck region: patients with chronic neck pain compared with pain-free persons. Arch Phys Med Rehabil 91:1878-1883, 2010

31. Tarnanen S, Neva MH, Dekker J, Häkkinen K, Vihtonen K, Pekkanen L, et al: Randomized controlled trial of postoperative exercise rehabilitation program after lumbar spine fusion: study protocol. BMC Musculoskelet Disord 13:123, 2012
32. Tarnanen S, Neva MH, Häkkinen K, Kankaanpää M, Ylinen J, Kraemer WJ, et al: Neutral spine control exercises in rehabilitation after lumbar spine fusion. J Strength Cond Res 28:2018-2025, 2014

33. Tarnanen S, Neva MH, Kautiainen H, Ylinen J, Pekkanen L, Kaistila T, et al: The early changes in trunk muscle strength and disability following lumbar spine fusion. Disabil Rehabil 35:134-139, 2013

34. Thorstensson A, Arvidson A: Trunk muscle strength and low back pain. Scand J Rehabil Med 14:69-75, 1982

\section{Disclosures}

The authors report no conflict of interest concerning the materials or methods used in this study or the findings specified in this paper.

\section{Supplemental Information \\ Current Affiliations}

Dr. Park: Department of Orthopedic Surgery, Samsung Medical Center, Sungkyunkwan University School of Medicine, Seoul, Republic of Korea.

Dr. Seo: Department of Orthopedic Surgery, Samsung Medical Center, Sungkyunkwan University School of Medicine, Seoul, Republic of Korea.

\section{Author Contributions}

Conception and design: Kang, Lee, Chung. Acquisition of data: Shin, Seo. Analysis and interpretation of data: all authors. Drafting the article: Kang, Lee, Park, Seo. Critically revising the article: Kang, Lee, Chung. Reviewed submitted version of manuscript: Kang, Chung, Park. Approved the final version of the manuscript on behalf of all authors: Kang. Statistical analysis: Kang, Shin.

\section{Correspondence}

Kyung-Chung Kang, Department of Orthopedic Surgery, Kyung Hee University Medical Center, 23 Kyungheedaero, Dongdaemun-gu, Seoul 130-872, Republic of Korea. email: futurespine@gmail.com. 